\title{
Creating Emotional Communication with Interactive Artwork
}

\author{
Matt Iacobini \\ University College \\ London, UCLIC \\ MPEB Gower Street \\ London, UK \\ m.iacobini@ucl.ac.uk
}

\author{
Tina Gonsalves \\ University College London \\ Wellcome Trust, \\ Neuroimaging Department \\ London, UK \\ tina@tinagonsalves.com
}

\author{
Nadia Bianchi Berthouze \\ University College London, \\ UCLIC \\ MPEB Gower Street London, \\ UK \\ n.berthouze@ucl.ac.uk
}

\author{
Chris Frith \\ University College Londo1 \\ Wellcome Trust \\ Department of Neuroimagir \\ London, UK \\ c.frith@ucl.ac.uk
}

\begin{abstract}
This pilot study contributes to the building of an art installation that aims to build an emotional communication loop with the audience. It will do so by reacting to or mimicking the audience's changes in emotional expressions according to emotional contagion dynamics. The study aims to inform the project by gaining a better understanding of emotional contagion patterns and the factors that may affect how people emotionally engage in this art context. The analysis of our early experiments shows reflex mechanisms of facial expression mimicry and countermimicry that follow patterns similar to those reported in the psychology literature. In fact, automatic mimicry and counter-mimicry correlated to some extent to whether or not the audience felt to be interacting with a real person. Furthermore, the results indicate that individual differences play a role in the way people can emotionally engage with this type of artwork. However, irrespective of these differences, the interaction led the audience to introspect and reflect about emotions.
\end{abstract}

\section{Introduction}

With emotion recognition technology becoming available $[1,2,3,7]$, it is now possible to create digital interactive art installations able to engage in an emotional interaction loop with their audience. These interactive emotion aware-art installations could have a wide variety of applications that go beyond art per-se, e.g., therapy. Various installations of this kind have been proposed with the intent to investigate the way people engage with them.

In [4], the interactive emotion-aware installation takes the form of a tree that recognizes the emotion of a person from voice and facial expressions. The tree grows in a naturalistic manner starting from an initial cluster of small shoots into a larger tree with coloured leaves. The user can influence this growth by interacting with the tree and creating a unique tree structure. The experiments showed that participants could strongly engage with the piece of art and were emotionally attached to their creation. However, differently from what the authors expected, their participants did not feel that the emotion expressed through the tree's emerging structure reflected their own emotions. The engaging experience was somehow built in a personal way through the interaction.

Wright et al. [5] presented an interactive artistic installation that creates an alter ego of the person it is interacting with, and displays emotions loosely mirroring those of that person, challenging their perception of identity. The participants communicated with the system, and found the experience very engaging and immersive.

Another interesting project is the Enactive Cinema project [6] where the spectator's heart rate is monitored to inform the character-based storyline. The way the narrative unfolds, the soundscape and the rhythm are biased by how the spectator experiences the emotional dynamics between the characters.

In [20], the art installation responds to its audience by expressing the audience's emotion using colours, shapes and meaning. The main idea is to invite the audience to emotionally interact with the installation by dynamically presenting them with visual representations of the captured audience's emotion or visual representation of different emotions.

The visual representations are created by capturing and automatically recognizing the facial expression of a person and then combining this expression with colours, shapes and messages selected from a database before projecting the expression back to the person. By realizing that the installation reacts to their expressions, the audience is led to act out different emotional expressions.

The study presented in this paper is part of the Chameleon project [8], another interesting project of this type. Through collaboration between artists, curators, neuroscientists, affective computing experts and human computer interaction teams, the Chameleon project investigates the scientific foundations of emotional contagion, transforming it into an art experience. It follows and critiques the scientific methodology, creating scientific and artistic research, as well as new models to be used in scientific experiments, and new ways to experience art.

The Chameleon installation interacts with its audience by recognizing the audience's emotional expressions and by displaying videos of emotional portraits (figure 1). It uses facial expression recognition technology [1] to detect the audience's emotional state and uses a database of emotional video portraits built by the artist to create the response.

Besides from creating an emotional bond with the audience and awareness of their emotional expressions 
as [20], our project also studies the more complex dynamics of emotional contagion [9], and ways to create emotional introspection in the audience. The main objective of the emotional portraits is hence to create an emotional communication loop: 1) by eliciting an automatic often unconscious emotional expression in the audience; and 2) by responding to the audience's emotional expression with an expression that reflects the emotional contagion process.

Before reporting on the results of the study, we briefly review the literature on emotional contagion.

\section{Emotional contagion}

Hatfield et al. [9] defined primitive emotional contagion as "the tendency to automatically mimic and synchronize facial expressions, vocalization, postures, and movements with those of another person and, consequently, to converge emotionally". The transfer of emotions is the product of a complex interaction between several processes, involving simulation of the other person's sensations and emotions, rationality, instinct, and conditioned reactions [19]. A possible neurological structure at the basis of this phenomenon is the mirror neuron system [11], the system at the basis of imitation.

Given the social role played by emotional expressions, it can be easily understood why such transfer mechanisms are very important in mediating social interaction. Various studies have shown that emotional contagion takes often place. In a study presented in [12], for example, participants were asked to listen to the voice of an actor reading an impartial script by using a sad, happy or neutral voice. Afterwards, when asked to rate their own emotions, the participants' emotion reflected the emotion of the actors they had listened to.

In [15], Hess and Blairy investigated whether people show emotional contagion in response to relatively weak and dynamic facial expressions of emotions of anger, sadness, happiness and disgust. They found evidence on mimicry for each type of emotion. Furthermore, they found evidence of emotional contagion for expressions of happiness and sadness but not for anger and disgust. In [21], facial electromyography was used to measure the responses of observers to facial expressions of sadness, fear, surprise, happiness, disgust and anger. The results showed that, to a large extent, the responses were consistent with the emotional expressions portrayed by the stimuli.

However, humans do not always respond to an emotional expression with exactly an equivalent emotional expression. Other evolutionary principles may guide social interaction. An expression of anger may unconsciously trigger an expression of sadness as a form of empathy. Gender and attitude towards the expressers are also factors that can affect the automatic response [16].

Furthermore, various studies (see [16] for a review) showed that the level of facial mimicry varies as a function of social context and type of emotional expression. In particular, it was found that whereas the mimicry of positive expressions was independent of the relation between observers and expressers, negative expressions were mimicked only when shown by a member of the group. Studies also showed that emotional expressions do not always trigger mimicry. In case of competition counter-mimicry is in fact observed, e.g., an expression of pain could trigger a smile [18].

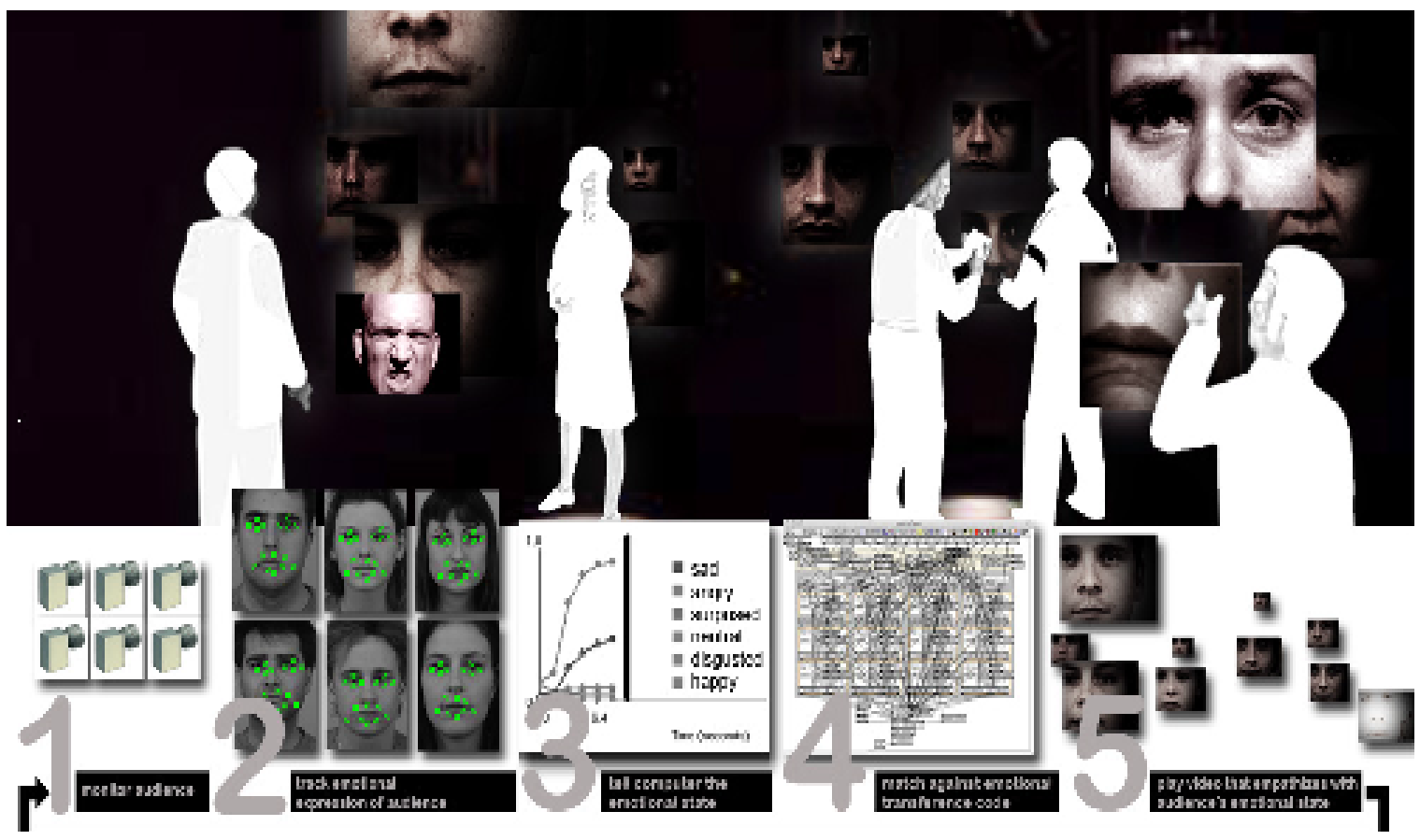

Figure 1: The Chameleon System 
Understanding, the dynamics that are at the basis of this emotional loop and exploit them to create emotional awareness is one of the aims of the Chameleon project. The paper is organized as follows. First, we introduce the research questions that this study has addressed. Then, section 4 describes the experiment setting and the experiment protocol used to address these questions. Finally we report and discuss the results of the experiment and of further interviews carried out with some of the participants.

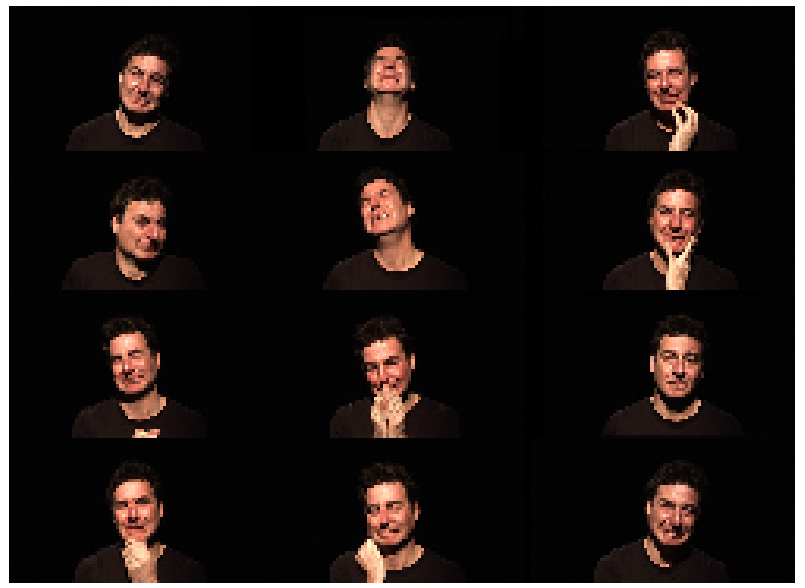

Figure 2: Examples of sad expressions from the Chameleon facial expression database.

\section{Research questions}

This study aims to investigate if and how people emotionally engage with videos of emotional portraits (see figure 2 for examples of video emotional portraits). In particular we are interested in creating a better understanding of: 1) the power that these emotional portraits have in inducing some form of unconscious emotional response in an observer; 2) the dynamics governing such responses; 3 ) the ability to induce a need for emotional introspection and reflection.

In order to address these questions, we have created a system that responds to the emotional expressions of an observer by displaying videos of emotional portraits selected from the Chameleon's database. The videos are selected on the basis of a set of rules (called here Emotion Transfer algorithm) built upon the principles of emotional contagion responses $[16,17,18]$ and on our general experience of emotional interaction. These rules are based upon probabilistic values associated to each type of emotional response. The probabilistic values are shown in table 1 . We do not argue that this set of rules reflects exactly the way humans react. The aim here is to create a form of human-like response (rather than a random or an exact replica) from the system and create the basis for exploring the existence of response patterns in the artwork context that may be integrated in later versions of the system.

\section{Experiment}

Since the Chameleon project is still in progress and the facial expression recognition system is not yet fully functional, we used a Wizard of Oz [13] type of scenario to investigate the questions.

A prototype was implemented on a laptop connected to a web cam and an external LCD screen (see Figure 3 for the set up of the experiment). The observer is sitting down at a desk and looking at an LCD screen showing a selection of videos of emotional expressions displaying six basic emotions (stimuli): happiness, sadness, anger, disgust, surprise, and neutrality. A web cam captures the face of the observer while s/he is looking at the stimulus. The wizard of $\mathrm{Oz}$ (called rater from now on) sits out of the line of sight of the observer, so as not to influence his/her emotions, and watches the observer through a computer connected to the web cam.

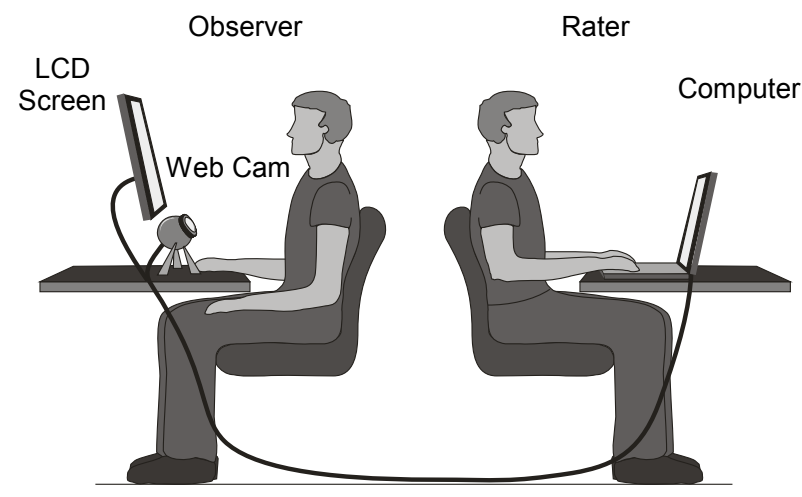

Figure 3: Wizard of $\mathrm{Oz}$ experiment set-up

The rater labels each change of facial expression of the observer in real time using one of six emotions categories. The label is automatically sent to the reaction engine that, by using the set of rules described in Table 1 , selects the new stimulus to present to the observer. The selection of the stimulus is made in such a way that the recurrences of the same video are minimized, i.e., a certain video is re-used only once all the instances of the videos of the same emotion expression category (e.g., happy) have been used. If the observer does not show any emotional response (i.e., a change in emotional expression), after 5 stimuli expressing the same emotion, have been shown, a new emotion category for the stimuli is randomly selected.

TABle 1 Emotion Transfer Algorithm’s Weights

\begin{tabular}{|c|c|c|c|c|c|c|}
\hline & \multicolumn{7}{|c|}{ Observers' Reactions } \\
\hline Stim. & Happy & Sad & Neutr. & Surpr. & Disg. & Angry \\
\hline Happy & $60 \%$ & $0 \%$ & $10 \%$ & $30 \%$ & $0 \%$ & $0 \%$ \\
\hline Sad & $0 \%$ & $70 \%$ & $20 \%$ & $0 \%$ & $0 \%$ & $10 \%$ \\
\hline Neutr. & $10 \%$ & $10 \%$ & $50 \%$ & $10 \%$ & $10 \%$ & $10 \%$ \\
\hline Surpr. & $15 \%$ & $15 \%$ & $5 \%$ & $50 \%$ & $5 \%$ & $10 \%$ \\
\hline Disg. & $0 \%$ & $0 \%$ & $0 \%$ & $25 \%$ & $60 \%$ & $15 \%$ \\
\hline Angry & $0 \%$ & $25 \%$ & $0 \%$ & $20 \%$ & $15 \%$ & $40 \%$ \\
\hline
\end{tabular}


The system keeps a time stamped record of the list of videos presented to the observer, of the observer's emotional responses (i.e. recorded video of his/her facial expression during the experiment) and of the rater's labels (i.e. the manual classification of the observers' emotional expressions). The experiment was split over 2 sessions of about 7 minutes each with a break of 3-5 min between them.

TABLE 2 RESUlTS FROM QUESTIONNAIRE AND NUMBER OF EXPRESSIVE REACTIONS (INCLUDING AND EXCLUDING NEUTRAL FACIAL EXPRESSION) TO STIMULI.

\begin{tabular}{|c|c|c|c|c|c|c|}
\hline \multirow[b]{2}{*}{ Observers } & \multicolumn{4}{|c|}{$\begin{array}{c}\text { Questionnaire } \\
\text { Responses } \\
\text { 1: strongly disagree, } \\
\text { 5: strongly agree }\end{array}$} & \multicolumn{2}{|c|}{$\begin{array}{c}\text { Number of } \\
\text { expressive reactions }\end{array}$} \\
\hline & Q1 & Q2 & Q3 & Q4 & $\begin{array}{c}\text { Inc. } \\
\text { Neutral }\end{array}$ & $\begin{array}{c}\text { Excluding } \\
\text { Neutral }\end{array}$ \\
\hline $\mathbf{O 0}$ & 4 & 4 & 2 & 3 & $\mathrm{X}$ & $\mathrm{X}$ \\
\hline 01 & 4 & 4 & 2 & 4 & 41 & 31 \\
\hline $\mathbf{O 2}$ & 3 & 4 & 3 & 2 & 44 & 35 \\
\hline $\mathbf{O 3}$ & 1 & 2 & 1 & 1 & 28 & 28 \\
\hline 04 & 5 & 4 & 3 & 4 & 66 & 60 \\
\hline 05 & 1 & 4 & 2 & 2 & 38 & 23 \\
\hline 06 & 4 & 4 & 4 & 3 & 35 & 35 \\
\hline $\mathbf{0 7}$ & 4 & 4 & 4 & 4 & 38 & 28 \\
\hline 08 & 2 & 2 & 3 & 3 & 50 & 35 \\
\hline O9 & 2 & 3 & 1 & 2 & 46 & 46 \\
\hline O10 & 4 & 4 & 4 & 2 & 50 & 40 \\
\hline Mean & 3.1 & 3.5 & 2.6 & 2.7 & 43.6 & 36.1 \\
\hline Mode & 4 & 4 & 2 & 2 & & \\
\hline
\end{tabular}

\subsection{Stimuli and participants}

The artist had created a database of short segments of video clips showing a mixture of actors and non-actors expressing emotions (Figure 2). Subjects were shot in a studio space with a black background. Twenty subjects were asked to express six basic emotional states: happiness, sadness, anger, disgust, surprise, and neutrality. A range of techniques were used to elicit emotional responses from the subjects. A subset of this database was used in this study: 5 different instances for each of the 6 categories of emotional expressions for 1 actor for a total of 30 different videos. Eleven observers were recruited on a voluntary basis amongst students and friends.

\subsection{Measuring the experience}

After the experiment all the observers were given a multiple choice form about their experience, and 5 of them were also interviewed. The possible answers for each question were on a 5-point scale: 1 - Strongly Disagree, 2 - Disagree, 3 - Neutral, 4 - Agree, 5 Strongly Agree. In this paper, we focus on the following four questions of the questionnaire:
Q1. I felt the emotion that the person on the screen was expressing.

Q2. I felt like I wanted to respond to the person on the screen

Q3. The interaction felt as if I was actually with another person

Q4. The interaction felt like when I am looking at the webcam feed of another person

\section{Analysis and Results}

Table 2 reports the results of the questionnaire and the reaction patterns of the observers.

The questionnaire results show that: 6 observers felt the stimulus emotions; 8 observers felt the desire to respond to the stimulus emotions; 5 observers felt that they were interacting with a person or looking at a person (according to the answers to Q3 and Q4). Observer $\mathrm{O} 5$ felt the desire to respond to the stimuli, although she said she did not experience their emotions.

According to the rater's classification, 10 observers reacted visibly during the experiment by displaying a series of different emotional expressions. Unfortunately, there was a problem with the recording of the first observer and the recording could not be stored. It is interesting to note that Observer O9 showed a frequency of expressive response above average even though he reported feeling neutral about wanting to respond. Instead, Observer $\mathrm{O} 3$ responded most negatively to the questionnaire and indeed reacted the least. However, a closer inspection of the recorded video of $\mathrm{O} 3$ reveals many reactions of lesser intensity that would not have been easily recognised by the rater in real time.

TABle 3: ReACtion PATterns of ObSERVERs: FOR EACH ENTRY OF THE TABLE, THE FIRST VALUE CORRESPONDS TO THE OBSERVERS THAT AGREED WITH QUESTION 3 (“... I FELT I WAS ACTUALLY WITH ANOTHER PERSON"), AND THE SECOND VALUE CORRESPONDS TO THE OBSERVERS THAT DISAGREED WITH IT.

\begin{tabular}{|c|c|c|c|c|c|c|}
\hline & \multicolumn{7}{|c|}{ Observers } \\
\hline Stim. & Happy & Sad & Neutr. & Surpr. & Disg. & Angry \\
\hline Happy & $\mathbf{7 3 \%}$ & $0 \%$ & $21 \%$ & $6 \%$ & $0 \%$ & $0 \%$ \\
& $\mathbf{8 4 \%}$ & $0 \%$ & $15 \%$ & $1 \%$ & $0 \%$ & $0 \%$ \\
\hline Sad & $7 \%$ & $\mathbf{6 5 \%}$ & $25 \%$ & $3 \%$ & $0 \%$ & $0 \%$ \\
& $2 \%$ & $\mathbf{4 4 \%}$ & $\mathbf{4 7 \%}$ & $6 \%$ & $0 \%$ & $0 \%$ \\
\hline Neutr. & $8 \%$ & $1 \%$ & $\mathbf{8 6 \%}$ & $4 \%$ & $0 \%$ & $0 \%$ \\
& $6 \%$ & $4 \%$ & $\mathbf{8 6 \%}$ & $3 \%$ & $0 \%$ & $0 \%$ \\
\hline Surpr. & $\mathbf{6 1 \%}$ & $0 \%$ & $13 \%$ & $26 \%$ & $0 \%$ & $0 \%$ \\
& $\mathbf{5 3 \%}$ & $0 \%$ & $24 \%$ & $20 \%$ & $3 \%$ & $0 \%$ \\
\hline Disg. & $19 \%$ & $4 \%$ & $\mathbf{6 3 \%}$ & $5 \%$ & $9 \%$ & $0 \%$ \\
& $\mathbf{4 6 \%}$ & $15 \%$ & $\mathbf{2 2} \%$ & $11 \%$ & $5 \%$ & $0 \%$ \\
\hline Angry & $1 \%$ & $28 \%$ & $\mathbf{4 2 \%}$ & $23 \%$ & $0 \%$ & $6 \%$ \\
& $0 \%$ & $4 \%$ & $\mathbf{8 6 \%}$ & $9 \%$ & $0 \%$ & $0 \%$ \\
\hline
\end{tabular}

One reason why some observers may have felt that they were not interacting with a person could be due to the fact that the used stimuli were mainly acted and 
sometimes exaggerated. It is possible that with more naturalistic expressions, the bond between observer and artwork might be easier to create.

Table 3 summarises the reaction patterns observed by the rater at run time. We have separated the results for the observers that felt they had been interacting with a person from those that felt they had not. The reason is that we expected different patterns of reaction between the two groups. In the rest of the paper, we call G1 the first group of observers and G2 the second group.

Comparing tables 1 and 3 , it can be seen that the observers reacted to the Happy, Sad and Neutral stimuli in a similar way to how the system is programmed to react to them. In particular, the clear mimicry patterns shown by both groups of observers $(73 \%, 80 \%)$ in response to Happy reflect the results reported in previous studies that positive facial expressions trigger mimicry and emotional contagion irrespective of the relation between observer and expresser and of the observer's attitude towards the expresser [16].

A similar but less pronounced instance of mimicry is seen for Sad. Whereas $65 \%$ of the G1 observers (i.e., that felt they were interacting with another person) mimicked the Sad expressions, only $44 \%$ of the G2 observers mimicked the expression and $47 \%$ remained Neutral. This is again in accordance with previous studies [16] that showed that in the case of negative emotion, a mimicry response can be inhibited or can elicit a counter-expression when observer and expresser are not socially related. Accordingly, Anger stimuli elicited mainly a Neutral reaction in both groups of observers, though G1 was more strongly affected, and reacted with more Sadness and Surprise. Disgust stimuli elicited mainly a Neutral response in G1 and mainly a Happy response in G2 (probably counter-mimicry of amusement). Surprise elicited mainly a Happy reaction in both groups (again to probably to do with amusement). The way the observers experience the interaction with the artwork may hence have an impact on their ability to empathize with it.

Overall these results indicate that the weights of the Emotion Transfer algorithm for negative emotions should be modified to take into account the relation that emerges between observer and expresser (audience and artwork). The Emotion Transfer algorithm should be endowed with a mechanism that can bias the selection of the response according to the relation that emerges between the observer and artwork. For example, counter-mimicry rules such as an expression of happiness in the observer in response to an expression of anger or disgust in the expresser (artwork) could be expected when a co-operative or empathic relation does not take place as shown in the study by Lanzetta and Englis [18]. The challenge hence becomes how to automatically detect or evaluate such relation. This question will be investigated in future work.

\subsection{Interviews: emotional introspection}

Five of the observers of the Wizard of $\mathrm{Oz}$ experiment were interviewed in a semi-structured manner. The results showed that a prolonged experience involving stimulus expressions perceived as emotionally congruent by the observer could indeed trigger reflection and introspection. One of the observers for example said: "I was thinking of some sad things that happened to me, when [...the stimulus] was sad for a while, it felt like a long time, and it reminded me of some things". Another observer reported: "I didn't like it when he looked sad and I didn't know why".

Also the vagueness of the interaction created space for reflection. An observer noted "I was looking at him and he turned around, it was strange, I asked myself why he would do that, it never happened to me, so I was looking at him to understand". Hence, situations that are stimulating on the one hand and vague on the other seem to facilitate engagement and reflection. They capture the attention of the observer, and induce him/her into a directed reflection by trying to clarify the meaning of the stimulus.

Another type of event that facilitated engagement with the artwork was behavioural mimicry. An observer reported that he felt that the videos was mimicking his gesture "If I did something with my hand he did it too". These events are consistent with Gaver et al. [14]. In fact, randomness introduced by errors, which is in itself meaningless, seems to generate a richer behaviour in people, as they assign meaning to it.

When asked about what diminished the quality of their experience, one of the observers said "when you are being filmed that's weird" and this made the experimenter notice in some of the following participants that the presence of the web cam was making them somewhat nervous. The level of engagement between observers and artwork could hence be increased by creating a more stimulating environment where people do not feel recorded. It is possible that in the real setting people would feel less observed as there will not be a rater evaluating them, but this will be automatically accomplished by the system in a more transparent way.

\section{Conclusions}

The aim of this study was to further understand the emotional contagion patterns that can occur when interacting with an artistic installation.

First of all, the experiments show that our composition of short video clips of actors expressing emotions is a compelling enough stimulus to elicit visible emotional reactions in the observers. All observers reacted visibly to the stimuli. Most observers reported that they felt the emotion expressed by the 
stimulus or the desire to react emotionally to it. These results are in line with the idea that reactions to emotional stimuli will arise with or without the perception, or intervention of the conscious part of the mind, as shown empirically in [10].

The reaction patterns to Happy, Sad and Neutral stimuli reflect the algorithm implemented in the system whereas Angry, Disgust and Surprise showed lack of mimicry or emotional contagion and the presence of counter-mimicry expressions. The level of mimicry and counter-mimicry correlated to whether the observer felt to be interacting with another person or not. This means that when undertaking such studies, an average across the whole result set may not represent a typical behaviour. It is hence necessary to conduct a larger scale study to identify self reportable traits that can predict the way the observers will relate in this sort of context.

The interviews with the observers gave evidence of introspective experiences, for example reflecting on events in their personal lives that they relate to the emotional stimuli. Even though the number of participants was small, the results indicate that such types of emotion-aware digital arts have the potential to affect people's emotions and hence to be used not only for the entertainment industry, but also in other situations such as therapy, where becoming aware of emotions in oneself and other people is an important step of the process.

At the time this paper is being written, we are investigating how these introspection effects persist with a large installation in a public environment, given the addition of audio, large screens and group interaction influencing the attention focus of the spectators in different proportions.

Furthermore, we are now using a wider variety of videos to improve the immersion and the general experience, after most people that were interviewed mentioned that repeating videos made it look "less real".

Overall, this preliminary experiment showed interesting and positive results. A more complete study that addresses the limitations discussed in this section needs however to be carried out to derive more definitive conclusions.

\section{References}

[1] R. el Kaliouby, P. Robinson. Real-time Inference of Complex Mental States from Facial Expressions and Head Gestures. Real-Time Vision for HCI, 181-200, Spring-Verlag, 2005.

[2] M. Pantic, A. Pentland, A. Nijholt, T.S. Huang. Human Computing and Machine Understanding of Human Behavior: A Survey. Artifical Intelligence for Human Computing, 47-71, 2007.

[3] N. Bianchi-Berthouze, A. Kleinsmith. A categorical approach to affective gesture recognition. Connection Science, 15 (4), 259-269, 2003.
[4] S.W. Gilroy, M. Cavazza, R. Chaignon, S.M. Mäkelä, M. Niranen, E. André, T. Vogt, J. Urbain, M. Billinghurst, H. Seichter, M. Benayoun. E-tree: emotionally driven augmented reality art. International Multimedia Conference Proceeding of the 16th ACM international conference on Multimedia, 945-948, 2008.

[5] A. Wright, E. Shinkle, and A. Linney. Alter Ego: Computer Reflections of human Emotions. Proceedings of the 6th Digital Art Conference, 2005.

[6] P. Tikka, R. Vuori, M. Kaipainen, J. Juutilainen. Enactive Cinema Project. Ongoing project refer to website http://www.enactivecinema.net/

[7] A. Camurri, M. Ricchetti, R. Trocca. EyesWeb - Toward Gesture and Affect Recognition in Dance/Music Interactive Systems. IEEE International Conference on Multimedia Computing and Systems, 1999.

[8] T. Gonsalves, C. Frith, H. Critchley, R. Picard, R. El Kaliouby. Chameleon. Ongoing project refer to website $\mathrm{http}: / /$ tinagonsalves.com/chamselected.htm.

[9] E. Hatfield, J. Cacioppo, R. L. Rapson. Emotional Contagion, Review of personality and social psychology. 14. Emotion and social behavior, pp. 151-177. Newbury Park, CA: Sage., 1992

[10] M. Tamietto, B. de Gelder. Emotional Contagion for Unseen Bodily Expressions: Evidence from Facial EMG. Proceeding of the FG 2008 meeting in Amsterdam, 2008.

[11] C. van der Gaad, R.B. Minderaa, C. Keysers. Facial expressions: What the mirror neuron system can and cannot tell us. Social Neuroscience, 2(3-4), 179-222, 2007

[12] R. Neumann, F. Strack. Mood contagion: The automatic transfer of mood between persons. Journal of Personality and Social Psychology, 79 (2), 211-223, 2000

[13] D. Maulsby, S. Greenberg, R. Mander. Prototyping an Intelligent Agent through Wizard of Oz. Proc ACM SIGCHI Conf. Human Factors in Computing Systems, ACM Press, 277-284, 1993.

[14] W. Gaver, J. Bowers, A. Boucher, A. Law, S. Pennington, and N. Villar. The History Tablecloth: Illuminating domestic activity, Proc. DIS 2006, 199 208.

[15] U. Hess, S. Blairy. Facial mimicry and emotional contagion to dynamic emotional facial expressions and their influence on decoding accuracy. International Journal of Psychophysiology, 40 (2), 129-141, 2001.

[16] P. Bourgeois, U. Hess. The impact of social context on mimicry. Biological Psychology, 77 (3), 343-352, 2008.

[17] W. Sato, S. Yoshikawa. Spontaneous facial mimicry in response to dynamic facial expressions. Cognition, 104(1), 1-18, 2007.

[18] J.T. Lanzetta, B.G. Englis, Expectations of Cooperation and Competition and Their Effects on Observers' Vicarious Emotional Responses. Journal of Personality and Social Psychology, 56 (4), 543-554, 1989.

[19] C. Frith, D. Wolpert. The Neuroscience of social Interaction. Decoding, Imitating, and Influencing the Actions of Others. Oxford University Press, 2003.

[20] I. Mihalinec, R. Stevanovic, K. Skala. Emotion Recognition and its Aesthetic Interpretation. IEEE 50th International Symposium on ELMAR, 2, 491-494, 2008.

[21] L. O. Lundqvist, Facial EMG reactions to facial expressions: a case of facial emotional contagion? Scandinavian Journal of Psychology, 36, 130-141, 1995. 